\title{
Experimental and computational methods for steel columns subjected to blast loading
}

\author{
L. K. Stewart \\ Department of Structural Engineering, University of California, \\ San Diego, USA
}

\begin{abstract}
The extreme environment generated by blasts that result from terrorist incidents can cause devastating consequences for structures and their occupants. Attacks in the form of vehicle bombs have motivated the necessity for designing and analysing structures to withstand these types of events. Typically, the development of such methodologies is driven by conclusions that have been obtained via field tests. Unfortunately, due to the harsh environment created by explosives, characterizing behaviours of structural components and collecting reliable data during a field blast event is problematic.

In an effort to provide answers to key questions related to how structures behave during a blast event and assuage the difficulty of producing high quality data, the University of California, San Diego (UCSD) Blast Simulator was developed. Using this technology, a series of experiments was conducted on fullscale structural steel columns to investigate the performance of the columns subjected to such vehicle borne threats. Because there is no fireball, high quality visual and quantitative data was produced which was used to validate the Simulator versus field testing and produce a high fidelity finite element model for column loading in both the weak and strong axis directions for future prediction needs.
\end{abstract}

Keywords: blast, steel, columns, simulator, finite element.

\section{Introduction}

The UCSD Blast Simulator [1] is the world's first hydraulically driven system that simulates blast-like loads on structures without the use of explosive materials. This is accomplished using an array of ultra-fast computer-controlled hydraulic actuators with a combined hydraulic/high pressure nitrogen energy 
source. An impulsive load is created by impacting the specimen with multiple impacting modules over a short duration.

Using this unique system, a series of steel column experiments were conducted to validate the Simulator for these types of structural systems and to produce both qualitative and quantitative data to develop computational tools for predicting blast response.

\section{Simulated blast loadings}

\subsection{UCSD blast simulator}

The Blast Simulator utilizes impacting modules that are accelerated to a prescribed velocity with an array of Blast Generators (BGs) shown in Figure 1. The BGs consist of a hydraulic actuator, control valves, accumulators, and transducers. Initially, nitrogen is compressed in the pressure accumulator along with high-pressured oil. A servo-controlled high-flow valve controls the oil flow into the actuator. Upon valve opening, the oil forces the piston rod/impacting mass assembly to drive outward toward the specimen. A smaller servo-controlled valve controls the outflow of the oil and thus is able to retract the actuator after impact. The force required to retract the actuator is supplied by pressurized nitrogen gas in a deceleration chamber, which is calibrated before each test.
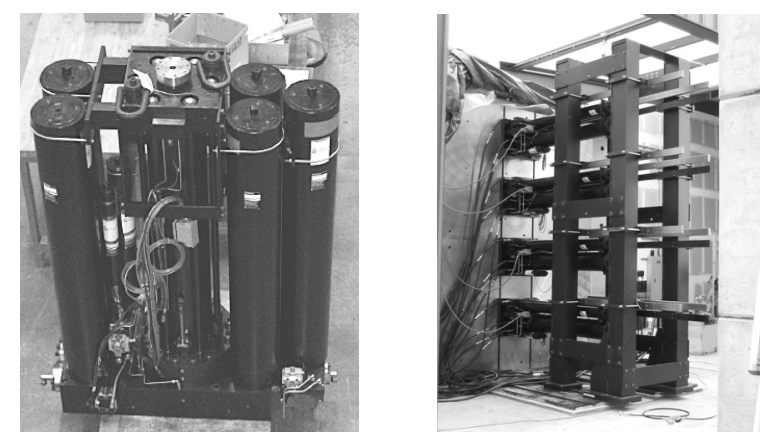

Figure 1: $\quad$ Blast generator (left) and BGs mounted to reaction wall (right).

The impacting module, which consists of a steel or aluminum mass, a thin aluminum backing plate, and a nonlinear, urethane material called a programmer, is used to transfer the energy and momentum of the module to the specimen. The programmer's geometric and material properties help tailor the duration and magnitude of the pressure, and thus the impulse to be representative of a blastlike pulse. The impacting modules are supported with guiderails that also align the modules with the specimen until impact. The rails are supported on a large frame system, which can be adjusted for various configurations.

The BGs are mounted to a fixed reaction wall, which is attached to a base isolated concrete slab. A moveable reaction wall is also attached to the slab and is used to mount specimens and fixtures. 


\subsection{Measurement techniques and methodology for blast simulator testing}

The initial velocity of each impacting module is determined from the desired impulse imparted on the specimen using eqn (1), where $I$ is the desired impulse, $m$ is the mass of the impacting module, $v$ denotes the change in velocity (impact velocity), and $A$ is the area over which the module is applied.

$$
I=\frac{m v}{A}
$$

The pressure and impulse applied to the specimen are derived from accelerometers located on the back of each impacting module shown in eqn (2) and (3), respectively. In the equations, $a(t)$ is the acceleration as a function of time, $p(t)$ is the pressure-time history and $I(t)$ is the impulse-time history.

$$
\begin{gathered}
p(t)=\frac{m a(t)}{A} \\
I(t)=\int p(t) d t
\end{gathered}
$$

The experiments are recorded using high-speed Phantom cameras, which record at 5,000 frames per second. Displacements throughout the duration of the tests are calculated from the video output using tracking software.

\section{Blast simulator experiments}

\subsection{Strong axis loading}

Three uniform velocity and eight variable velocity tests were conducted on five strong axis column specimens loaded in the strong axis direction. A summary of these tests with desired target BG impact velocities is given in Table 1.

Table 1: $\quad$ Strong axis loading test matrix.

\begin{tabular}{|c|c|c|c|c|}
\hline Test & Specimen & Column & Loading & Desired Impact Velocity \\
\hline SA-01 & 1 & W10x49 & Uniform & $157.5 \mathrm{in} / \mathrm{s}(4.0 \mathrm{~m} / \mathrm{s})$ \\
\hline SA-02 & 1 & W10x49 & Uniform & $708.6 \mathrm{in} / \mathrm{s}(18.0 \mathrm{~m} / \mathrm{s})$ \\
\hline SA-03 & 2 & W10x49 & Uniform & $1023.6 \mathrm{in} / \mathrm{s}(26.0 \mathrm{~m} / \mathrm{s})$ \\
\hline SA-04 & 3 & W14x132 & Variable & $\begin{array}{c}391.7,325.5,267.7,226.4 \mathrm{in} / \mathrm{s} \\
(9.9,8.3,6.8,5.8 \mathrm{~m} / \mathrm{s})\end{array}$ \\
\hline SA-05 & 3 & W14x132 & Variable & $\begin{array}{c}389.4,325.5,267.7,226.4 \mathrm{in} / \mathrm{s} \\
(9.9,8.3,6.8,5.8 \mathrm{~m} / \mathrm{s})\end{array}$ \\
\hline SA-06 & 3 & W14x132 & Variable & $\begin{array}{c}377.6,322.8,266.5,224.4 \mathrm{in} / \mathrm{s} \\
(9.6,8.2,6.8,5.7 \mathrm{~m} / \mathrm{s})\end{array}$ \\
\hline SA-07 & 3 & W14x132 & Variable & $\begin{array}{c}1744.1,1389.8,1003.9,502.9 \mathrm{in} / \mathrm{s} \\
(44.3,35.5,25.5,12.8 \mathrm{~m} / \mathrm{s})\end{array}$ \\
\hline SA-08 & 4 & W14x132 & Variable & $\begin{array}{c}320.5,264.6,213.8,181.1 \mathrm{in} / \mathrm{s} \\
(8.1,6.7,5.4,4.6 \mathrm{~m} / \mathrm{s})\end{array}$ \\
\hline SA-09 & 4 & W14x132 & Variable & $\begin{array}{c}175.9,1401.6,1007.9,507.9 \mathrm{in} / \mathrm{s} \\
(44.6,35.6,25.6,12.9 \mathrm{~m} / \mathrm{s})\end{array}$ \\
\hline SA-10 & 5 & W14x132 & Variable & $\begin{array}{c}1779.5,1425.2,1031.5,511.8 \mathrm{in} / \mathrm{s} \\
(45.2,36.2,26.2,13.0 \mathrm{~m} / \mathrm{s})\end{array}$ \\
\hline SA-11 & 1 & W10x49 & Variable & $\begin{array}{c}1594.5,1334.6,1267.7,972.4 \mathrm{in} / \mathrm{s} \\
(40.5,33.9,32.3,24.7 \mathrm{~m} / \mathrm{s})\end{array}$ \\
\hline
\end{tabular}




\subsubsection{Test specimens}

The W14x132 strong axis specimens used in this test series were identical to columns used in field tests. Two additional W10x49 specimens were constructed to provide additional data. The specimens were $10.75 \mathrm{ft}(3.3 \mathrm{~m})$ in clear height and $15.5 \mathrm{ft}(4.7 \mathrm{~m})$ in total height with the header and footer. Each column specimen had a concrete header, which prevented rotation at the top of the column. The top of the column was welded to a 36 in by 36 in by 0.5 in steel plate. The four sides of the header were confined by 0.25 in steel plates, which created a $3 \mathrm{ft}$ cube that filled with 5,000 psi concrete.

The footer was designed in the same manner consisting of a $3 \mathrm{ft}$ by $3 \mathrm{ft}$ by $1.75 \mathrm{ft}$ cube of 5,000 psi concrete.

\subsubsection{Test setup}

The test setup is illustrated in Figure 2. The columns were loaded with four blast generators over the height of the column. For the uniform tests, four BG $25 \mathrm{~s}$ were used and two BG 25s and two BG 50s were used for the variable tests. The BGs are numbered 1 to 4 with 1 being the bottom BG and 4 being the top.
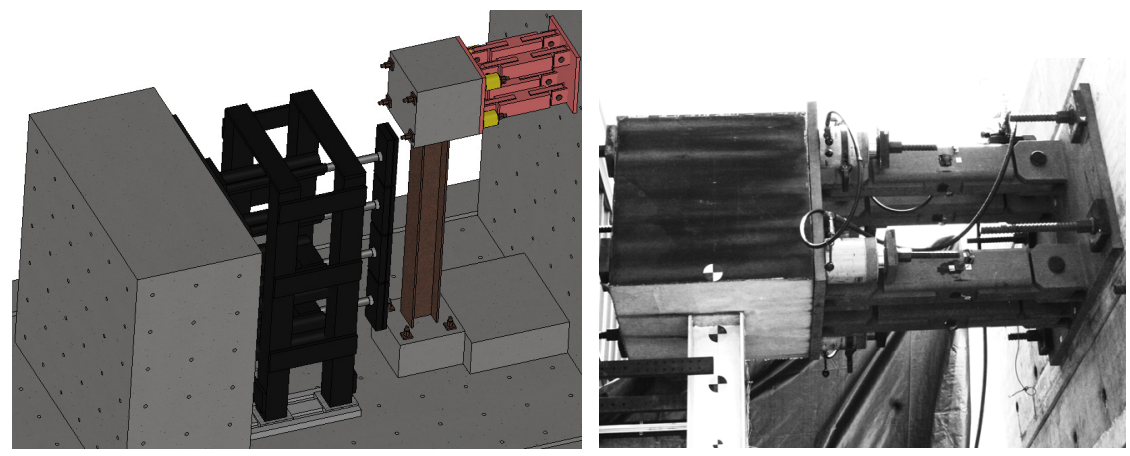

Figure 2: $\quad$ Test setup (left) with upper link boundary condition (right).

The impacting modules varied in dimension for the two size specimens. For the W10x49 experiments, the four impacting units were 10 in wide by 30 in tall. The impacting modules included a 3.75 in thick aluminum impacting mass and a 2 in thick programmer. The W14x132 experiments used four impacting modules that were 14 in by 30 in. The impacting modules included a 3.25 in thick steel impacting mass and a 2 in thick programmer.

The boundary conditions for the tests were selected because they were similar to the actual behavior of a building column subjected to blast loads and to conditions applied during field tests to which data could be compared. The connection at the base of the column was restrained in all directions simulating a "fixed" condition. It was post-tensioned to the reaction floor and a concrete spacer block transferred the shear to the reaction wall. The header was attached to a link system, shown in Figure 2, which allowed the column to move vertically while providing lateral and moment restraint. The link system was 
post-tensioned to the load stub and reaction wall. Four hollow hydraulic jacks tensioned the load stub header to the link and were left in place during the test.

\subsubsection{Test results}

The results from one representative strong axis column tests will be included in this section the additional results can be found in detail in [2].

\subsubsection{Test SA-10 results}

Test SA-10 was conducted on Specimen 5 and was considered a high-velocity test relative to the other experiments. Figure 3 shows the progression of damage recorded by the high-speed camera. In (a), the BGs are set to the initial position. The impact is shown in (b) and the synchronicity of the BGs can be seen. Figures (c) through (f) capture the propagation of deformation of the column while the masses are in contact with the column. (g) displays the deformation of the specimen after the masses have retracted.

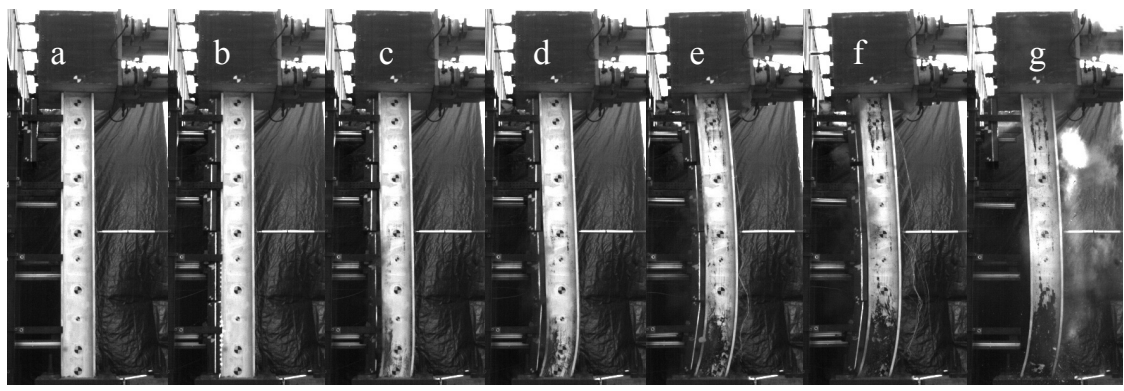

Figure 3: $\quad$ Test SA-10 progression of damage from high-speed camera.

Table 2 gives a summary of (unfiltered) peak acceleration, impact time, duration, and impulse for each BG as well as the impact velocities, which were computed from the data from the Phantom cameras.

Table 2: $\quad$ Test SA-10 summary of BG impact details.

\begin{tabular}{|c|c|c|c|c|c|}
\hline BG & $\begin{array}{c}\text { Peak } \\
\text { Acceleration } \\
(\mathrm{g})\end{array}$ & $\begin{array}{c}\text { Impact Time } \\
(\mathrm{ms})\end{array}$ & $\begin{array}{c}\text { Duration of } \\
\text { Contact } \\
(\mathrm{ms})\end{array}$ & $\begin{array}{c}\text { Impact } \\
\text { Velocity } \\
\text { in/s (m/s) }\end{array}$ & $\begin{array}{c}\text { Calculated Impulse } \\
\text { from Accelerometer } \\
\text { psi-ms (MPa-ms) }\end{array}$ \\
\hline 1 & 23,470 & 51.06 & 1.81 & $1,925.0(48.9)$ & $7,796(53.8)$ \\
\hline 2 & 13,860 & 50.91 & 2.02 & $1,621.5(41.2)$ & $3,600(24.8)$ \\
\hline 3 & 1,300 & 50.28 & 2.97 & $1,117.3(28.4)$ & $3,300(22.8)$ \\
\hline 4 & 1,360 & 50.83 & 4.25 & $530.4(13.5)$ & $2,192(15.1)$ \\
\hline
\end{tabular}

\subsection{Weak axis loading}

One uniform velocity and five variable velocity tests were conducted on six weak axis specimens. A summary of these tests with desired target BG impact velocities and loading medium (described in Section 3.2.2) is given in Table 3. 
Table 3: $\quad$ Weak axis loading test matrix.

\begin{tabular}{|c|c|c|c|c|c|}
\hline Test & Specimen & Column & Loading & Medium & Desired Impact Velocity \\
\hline WA-01 & 6 & W10x49 & Uniform & Sand & $1102.4 \mathrm{in} / \mathrm{s}(28 \mathrm{~m} / \mathrm{s})$ \\
\hline WA-02 & 7 & W14x132 & Variable & Water & $\begin{array}{c}1574.8,1299.2,984.3,629.9 \mathrm{in} / \mathrm{s} \\
(40.0,33.0,25.0,16.0 \mathrm{~m} / \mathrm{s})\end{array}$ \\
\hline WA-03 & 8 & W14x132 & Variable & Water & $\begin{array}{c}1732.3,1456.7,1003.9,708.7 \mathrm{in} / \mathrm{s} \\
(44.0,37.0,25.5,18.0 \mathrm{~m} / \mathrm{s})\end{array}$ \\
\hline WA-04 & 9 & W14x132 & Variable & Sand & $\begin{array}{c}1732.3,1456.7,1003.9,708.7 \mathrm{in} / \mathrm{s} \\
(44.0,37.0,25.5,18.0 \mathrm{~m} / \mathrm{s})\end{array}$ \\
\hline WA-05 & 10 & W10x49 & Variable & Water & $\begin{array}{c}2362.2,1870.1,1378.0,855.8 \mathrm{in} / \mathrm{s} \\
(60.0,47.5,35.0,22.5 \mathrm{~m} / \mathrm{s})\end{array}$ \\
\hline WA-06 & 11 & W10x49 & Variable & Sand & $\begin{array}{c}2362.2,1870.1,1378.0,855.8 \mathrm{in} / \mathrm{s} \\
(60.0,47.5,35.0,22.5 \mathrm{~m} / \mathrm{s})\end{array}$ \\
\hline
\end{tabular}

\subsubsection{Test specimens}

The specimens used for the weak axis test series were identical to those for the strong axis tests described in Section 3.1.1 with the exception of the column being rotated 90 degrees.

\subsubsection{Test setup}

The test setup for the weak axis tests utilized the same BG configuration and boundary conditions as the strong axis tests discussed in Section 3.1.2.

In order to sufficiently load the weak axis, pressure must be imparted on both the column web and along the insides of both column flanges. This cannot be done using a flat programmer as was done in the previous tests.

Research by Huson et al. [3] demonstrated the use of water filled bladders as a loading medium to transfer the pressure and impulse to all inner surfaces. Both sand and water were used in these experiments in order to achieve a range of responses to simulate various structural scenarios such as the use of cladding. Custom made bladders were produced and filled with the appropriate material and installed in the inside of the columns.

The bladders were loaded with the impacting modules, which varied in dimension. For the W10x49 tests, the four impacting units were 6 in by 30 in. The impacting units included 4 in thick aluminum impacting mass and a 2 in thick programmer. The W14x132 tests used four impacting modules that were 10 in by 30 in. The units included a 4.5 in steel impacting mass and a programmer.

\subsubsection{Test Results}

The results from column tests will be included in this section from a W10x49 column, which used sand as the medium (Test WA-06). Additional results can be found in detail in [2].

3.2.3.1 Test WA-06 results Test WA-06 was a variable velocity impact with sand as the loading medium to simulate a column without cladding. Figure 5 gives the results from the high-speed camera. In (a), the BGs are set to the correct initial position. The initial impact is shown in (b). Figures (c) through (f) capture the propagation of deformation of the column while the masses are loading the bladder and (g) displays the deformation of the specimen post test. 


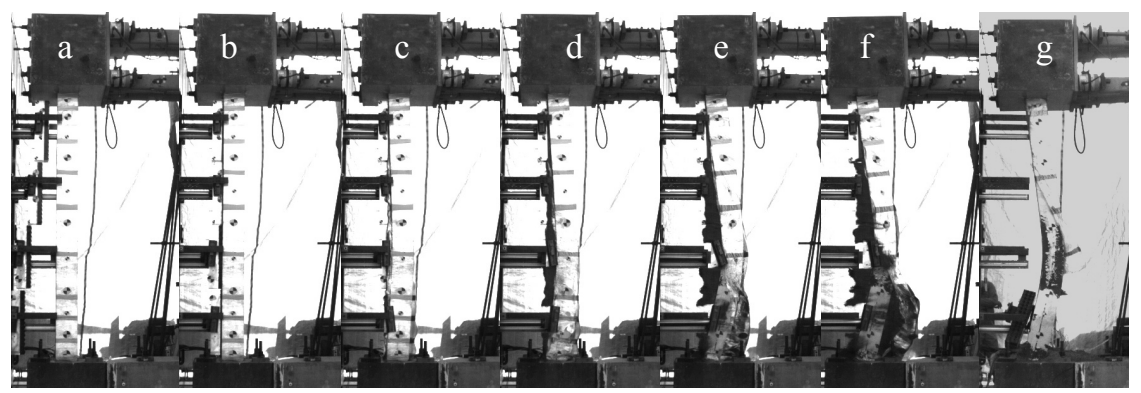

Figure 5: Test WA-06 progression of damage from high-speed camera.

Table 4 gives a summary of peak acceleration, impact time and duration for each BG as well as the impact velocities, which were computed from the data from the Phantom cameras and the calculated applied impulse to the bladder. Displacements and qualitative comparisons to finite element calculations are given in Section 5.

Table 4: $\quad$ Test WA-06 summary of BG impact details.

\begin{tabular}{|c|c|c|c|c|c|}
\hline BG & $\begin{array}{c}\text { Peak } \\
\text { Acceleration } \\
(\mathrm{g})\end{array}$ & $\begin{array}{c}\text { Impact Time } \\
(\mathrm{ms})\end{array}$ & $\begin{array}{c}\text { Duration of } \\
\text { Contact } \\
(\mathrm{ms})\end{array}$ & $\begin{array}{c}\text { Impact } \\
\text { Velocity } \\
\text { in/s }(\mathrm{m} / \mathrm{s})\end{array}$ & $\begin{array}{c}\text { Calculated Impulse } \\
\text { from Accelerometer } \\
\text { psi-ms (MPa-ms) }\end{array}$ \\
\hline 1 & $3,231.6$ & 37.34 & 7.94 & $25,53.7(64.9)$ & $15,584(107.4)$ \\
\hline 2 & $1,900.5$ & 43.82 & 21.54 & $2,050.7(52.1)$ & $12,456(85.9)$ \\
\hline 3 & $1,673.7$ & 42.03 & 12.86 & $1,273.9(32.6)$ & $8,523(58.8)$ \\
\hline 4 & 925.4 & 41.15 & 7.19 & $840.3(21.3)$ & $5,678(39.4)$ \\
\hline
\end{tabular}

\section{Computational model}

A finite element model (FEM) for the steel columns under simulated blast loads was developed for use as a tool for blast response prediction. The finite element analysis was preformed with LS-DYNA, a three dimensional, explicit, Lagrangian finite element code.

\subsection{Strong axis loading LS-DYNA model}

Six strong axis tests with the highest impulse were modeled using the LS-DYNA mesh shown in Figure 6. The model was calibrated with the data from the simulator tests and comparisons are shown in Section 5.1.

\subsubsection{Column specimen}

The steel column was modeled with fully integrated shell elements with six integration points through the thickness. To model the steel material *MAT_PIECEWISE_LINEAR_PLASTICITY was used. This model allows for the input of arbitrary stress versus strain relations for various strain rates. The values for the stress-strain relations at each given rate were found through many rounds of experimental testing. 

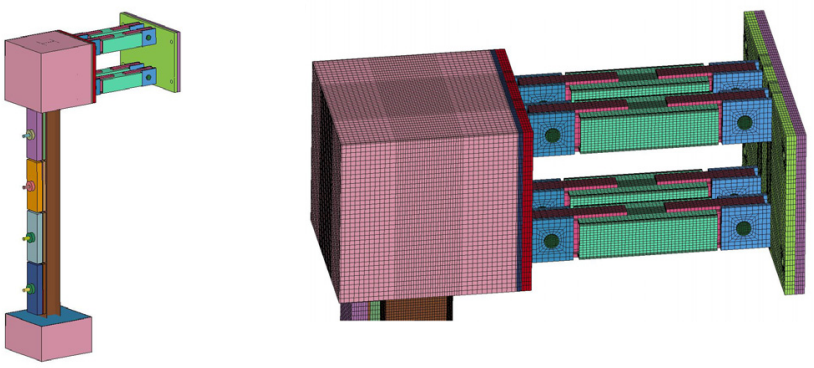

Figure 6: LS-DYNA model of strong axis column (left) and link system (right).

The concrete header and concrete footer model used three dimensional brick elements with single point integration. The concrete was modeled with the K\&C Concrete Model, *MAT_072 Rel 3 [4]. To account for strain rate effects, tabulated data was used determined from calibrated test data provided by $\mathrm{K} \& \mathrm{C}$.

\subsubsection{Boundary conditions}

The boundary conditions on the footer were prescribed such that the nodes on the base were restrained in all directions, simulating the fixed condition. Initially, to model the header, it was assumed that the system remained fixed and the rotation and was only allowed to translate vertically and could be modeled using simple nodal restraints; however, at the higher-level velocity tests, these boundary conditions were not fully met. The system began to act more like a pin connection than a fixed connection as the velocities increased.

To incorporate this, the entire link system was modeled as shown below in Figure 6 and connected to a steel backing plate. The backing plate had a fixed restraint on the back of one side and was merged to the tabs, which hold the pins on the other. For both sets of pins, a contact surface was placed between the pin and the surrounding parts to allow rotation. To correctly model the extra rotation for higher velocity tests, a layer of calibrated rubber was placed between the link and the header. The layer allowed for the link to be stiffer at small displacements as seen in the low-velocity impacts, while displacing more for the high-velocity.

\subsubsection{Loading}

Loads were applied to the column by modeling the impacting module with an initial velocity. The rod was not fully modeled, but the density of the material was changed include the full mass of the rod.

A programmer model was developed by Oesterle [5] to simulate a testing series on concrete masonry walls such that the material could impact the wall through a contact surface applied through the face of the wall and the face of the programmer material. The model chosen was *MAT_LOW_DENSITY_FOAM because of the similarities between the stress-strain behavior and the energy dissipation characteristics between low-density foam and the programmer. 


\subsubsection{Finite element model results}

The finite element model results from Test SA-10 are shown in Figure 7.
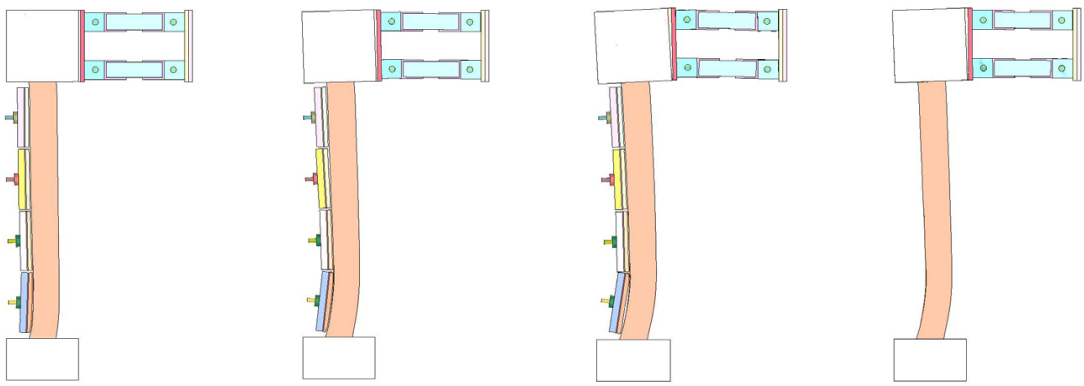

Figure 7: $\quad$ Test SA-10 finite element model.

\subsection{Weak axis loading LS-DYNA model}

The weak axis model was identical to the strong axis model with the exception of the loading. For the sand experiments, Smooth Particle Hydrodynamics (SPH) elements were used to model the sand in the inner part of the column. The sand model used was *MAT_GEOLOGICAL_CAP_MODEL, which was calibrated from [6]. The water tests were modeled by applying a pressure-time history along the column height on both the column web and the inner flanges. These pressure-time histories were computed using the hydrodynamics code, CTH.

\subsubsection{Finite element model results}

The finite element model result from Test WA-05 is shown in Figure 8.

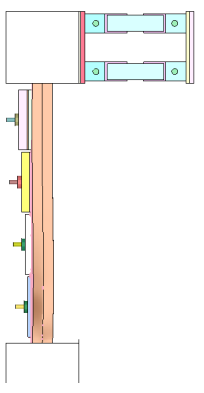

Residual
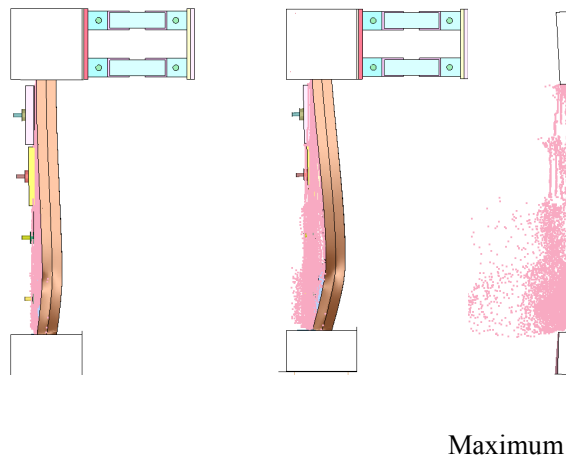

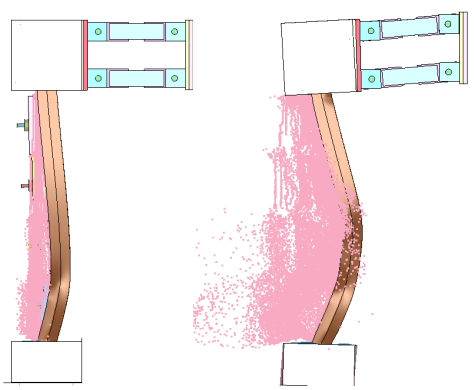

Maximum

Figure 8: $\quad$ Test WA-05 finite element model results.

\section{Comparisons}

Comparisons to field test data were preformed whenever possible. Identical test data was available for the W14x132 specimens in the strong axis direction only. The W10x49 field test data was available for similar tests therefore only 
qualitative comparisons were made. Comparisons were made between the simulated experiments and the computational model for all tests performed.

\subsection{Strong axis loading}

Residual displacements along the height of the column were used to compare the behavior of the simulated experiments with that of the live field experiments. An example of this is given in Figure 9 from Test SA-10. This comparison shows good correlation between the two methods of loading.
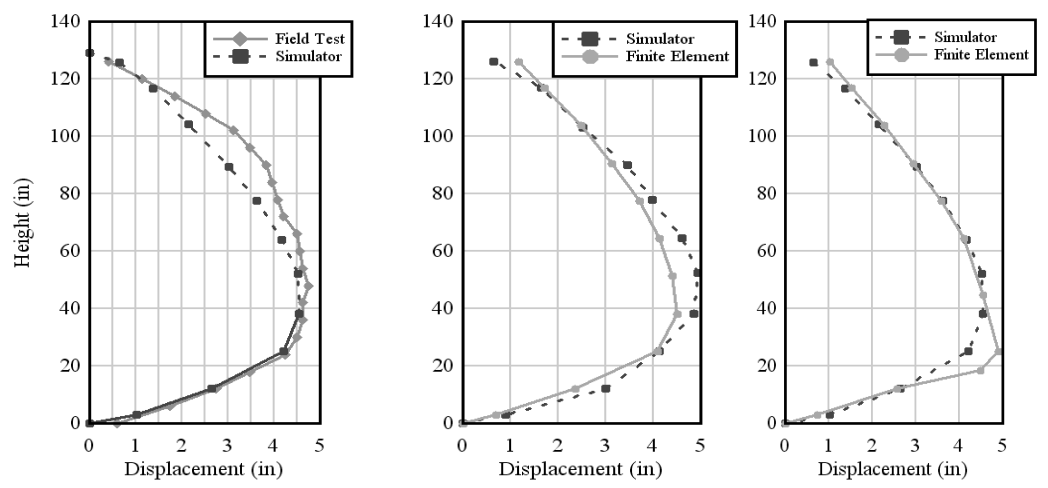

Figure 9: Residual and maximum displacement comparison for strong axis.

The maximum displacements along the height of the column were used to compare the results from the simulated experiments and the finite element model. Results from two tests (Test SA-09 and Test SA-10) with moderate to high impact velocities are also shown in Figure 9.

Qualitatively, comparisons can be made for both global column behaviors and localized column blast response. Some observations from the strong axis tests are shown. Figure 10 show a global comparison of column deformation from Test SA-11. Figure 11's pictures show a comparison of localized buckling, which occurred at the base of the column in the same test. The finite element model is able to sufficiently capture both column responses adequately.
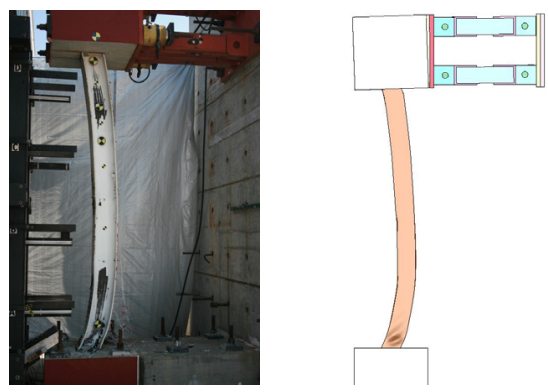

Figure 10: Global deformation comparisons for test SA-11. 

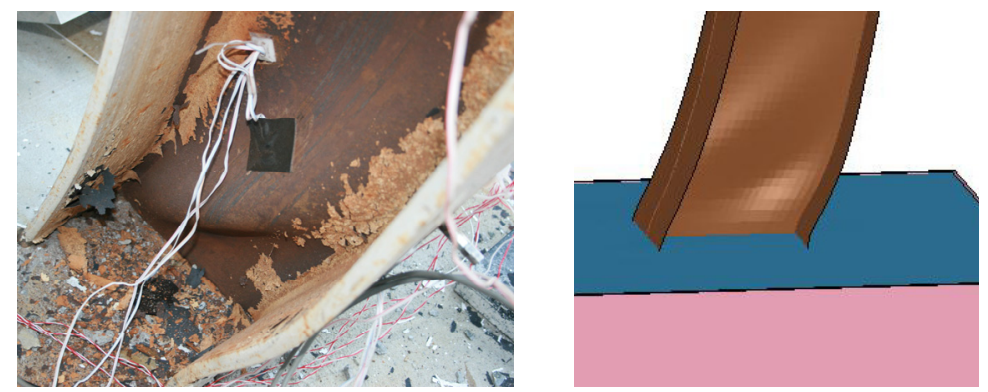

Figure 11: Local buckling comparisons for test SA-11.

\subsection{Weak axis loading}

The maximum displacements were used to compare the results from the simulated experiments and the finite element model. Results from all of the six tests show results consistent with those in the strong axis tests shown above.

Qualitatively, comparisons can be made between the field tests, simulated tests and the finite element models. Figure 12 pictures are from Test WA-05, which corresponds to a field test with cladding. Figure 13 shows pictures
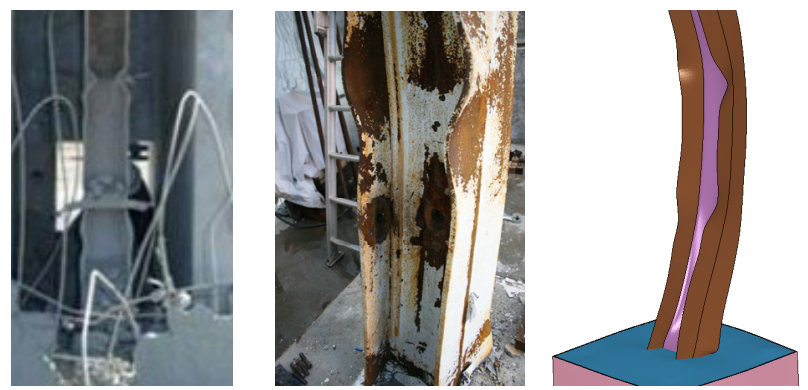

Figure 12: Field, simulator, and FEM comparisons for column with cladding.
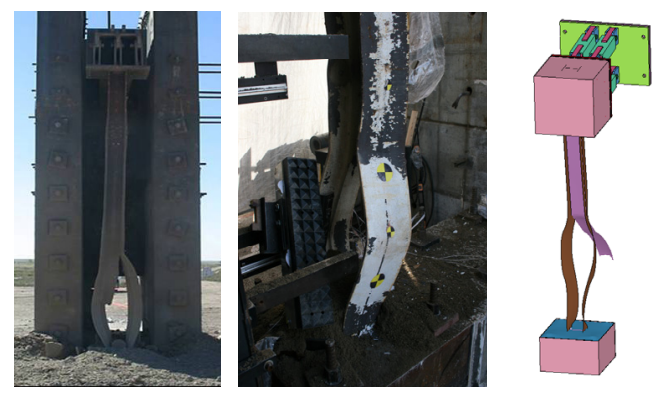

Figure 13: Field, simulator, and FEM comparisons for column without cladding. 
correspond to Test WA-06 and a field test without cladding. The finite element model does a sufficient job of capturing the response of the column, with the exception of the full web separation for which a coupled model would be needed to capture the venting effects through the hole that is formed.

\section{Conclusions}

Through testing of multiple steel columns with various configurations and comparing them to live explosive field experiments, the UCSD Blast Simulator was shown to be an effective method for applying simulated blast load on steel columns. The Simulator experiments provided data used to develop a finite element model which was able to predict accurate response to blast loadings.

\section{References}

[1] Hegemier, G., Seible, F., Arenett, K., Rodriguez-Nikl, T., Oesterle, M., Wolfson, J., Gram, M., and Clark, A., The UCSD Blast Simulator. $77^{\text {th }}$ shock and Vibration Symposium. Monterey, CA, 2006.

[2] Stewart, L., Testing and Analysis of Structural Steel Columns Subjected to Blast Loads, La, Jolla, CA. 2010.

[3] Huson, P., Asaro, R., Stewart, L., and Hegemier, G., Non-explosive methods for simulating blast loading on structures with complex geometry. Impact Engineering, 38(7), pp. 546-557, 2011.

[4] Malvar, L., Crawford, J., Wesevich, J., and Simons, D., A Plasticity Concrete Material Model of DYNA3S. Impact Engineering. 19(9-10), pp. 847-873, 1997.

[5] Oesterle, M., Blast Simulator Wall Tests: Experimental Methods and Mitigation Strategies for Reinforced Concrete and Concrete Masonry, La Jolla, CA, 2009.

[6] Green, M., Sheer Friction Test Support Program: Laboratory Test Results for WES Flume Sand Backfill. Vicksburg, MS 1984. 